\title{
A Pilot Randomized Controlled Trial of Early versus Routine Caffeine in Extremely Premature Infants
}

\author{
Anup C. Katheria, $\mathrm{MD}^{1}$ Jason B. Sauberan, PharmD \\ Jayson Durham, BA ${ }^{1}$ Neil N. Finer, MD ${ }^{1,2}$ \\ ${ }^{1}$ Neonatal Research Institute, Sharp Mary Birch Hospital for Women \\ and Newborns, San Diego, California \\ ${ }^{2}$ Department of Pediatrics, University of California, San Diego, \\ California
}

\author{
Devang Akotia, BS ${ }^{1}$ Wade Rich, BS, RRT ${ }^{1}$
}

\begin{abstract}
Address for correspondence Anup C. Katheria, MD, Neonatal Research Institute, Sharp Mary Birch Hospital for Women and Newborns, 3003 Health Center Drive, San Diego, CA 92123 (e-mail: anup.katheria@sharp.com).
\end{abstract}

Am J Perinatol 2015;32:879-886.

\begin{abstract}
Keywords

- caffeine

- hemodynamics

- intubation

- vasopressors
\end{abstract}

Respiratory distress syndrome can be successfully treated in very preterm infants with nasal continuous positive airway pressure (CPAP) beginning at birth. ${ }^{1}$ However, many of these infants will fail CPAP and require endotracheal intubation and surfactant administration to achieve adequate gas exchange. ${ }^{2,3}$ Early use of caffeine as a respiratory stimulant represents a potential adjunctive therapy with CPAP to prevent intubation.

received

October 27, 2014

accepted after revision

December 5, 2014

published online

January 21, 2015

In the Caffeine for Apnea of Prematurity (CAP) trial, infants in the caffeine group were able to discontinue positive pressure ventilation approximately 1 week earlier than the placebo group, and had a significant reduction in their incidence of bronchopulmonary dysplasia (BPD). ${ }^{4}$ The average postnatal age of caffeine initiation in the CAP trial was 3 days. Posthoc analyses of the CAP trial, and retrospective studies performed by other groups, have discovered

Copyright $\odot 2015$ by Thieme Medical Publishers, Inc., 333 Seventh Avenue, New York, NY 10001, USA. Tel: +1(212) 584-4662.
DOI http://dx.doi.org/ 10.1055/s-0034-1543981. ISSN 0735-1631. 
reductions in respiratory and neurologic morbidities associated with earlier caffeine initiation. ${ }^{5}$

Few, small, observational studies have investigated the cardiovascular effects of caffeine in the neonate. Some have demonstrated transient decreases in cerebral and intestinal blood flow after a caffeine dose with no change in cardiac output. ${ }^{6}$ Others have shown increased blood pressure after a dose of intravenous caffeine. ${ }^{7,8}$ It is possible that these cardiovascular effects could be beneficial for early transitional circulation, especially in the premature infant who is prone to hypotension and cardiac dysfunction.

Recently, some European centers have adopted a technique to administer surfactant via a thin flexible catheter during spontaneous breathing while receiving nasal CPAP (minimally invasive surfactant therapy). ${ }^{9}$ As part of this protocol, infants are given intravenous caffeine in the 1st hour of age. ${ }^{10}$ Other European centers have adopted the use of early caffeine as soon as infants have intravenous access along with antibiotics and fluids (Jan Mazela, MD, PhD, verbal communication, May 2014). However, this practice has never been demonstrated to be beneficial in a randomized controlled trial. Our center has a range of caffeine initiation from 2 to 24 hours of age.

We hypothesized that very early caffeine given in the first 2 hours after birth to nonintubated preterm infants on nasal CPAP will be associated with a reduction in risk of intubation within the first 12 hours of age, and with improved measures of systemic blood flow, compared with routine caffeine administration at 12 hours of age (our center's average time of first administration). Given the lack of previously published studies, our objective was to perform a pilot trial to determine the appropriate power for a larger study.

\section{Study Design}

This double blinded, randomized, placebo-controlled pilot study was conducted in the level III neonatal intensive care unit (NICU) at the Sharp Mary Birch Hospital for Women and Newborns in San Diego, CA between September 2013 and May 2014. The study was approved by the Sharp HealthCare Institutional Review Board, and registered with ClinicalTrials. gov (NCT 01783561). A waiver of delayed consent was approved for infants where adequate time was not available for antenatal consent. Informed written consent was obtained before any study-related echocardiographic or laboratory data collection.

Any newborn delivered between 23 and $28^{6 / 7}$ weeks gestational age (GA) by best obstetric estimates was eligible for inclusion. Exclusion criteria were one or more of the following: (1) major congenital anomaly including airway anomalies, congenital diaphragmatic hernia, or hydrops, (2) known or a discovered major cardiac defect other than a patent ductus arteriosus (PDA), patent foramen ovale (PFO), or small ventricular septal defect, (3) inability to have a peripheral or central catheter placed within the first 60 minutes of age, and (4) severe apnea or bradycardia in the first 10 minutes of age requiring emergent endotracheal intubation.
Eligible neonates were randomly assigned to receive intravenous caffeine citrate $20 \mathrm{mg} / \mathrm{kg}$ infused over 15 minutes within the first 2 hours of age and then placebo (normal saline) of identical volume and appearance at 12 hours of age (early caffeine group), or placebo in the first 2 hours of age and intravenous caffeine $20 \mathrm{mg} / \mathrm{kg}$ infused over 15 minutes at 12 hours of age (routine caffeine group). In both groups, a single blood sample was obtained at 13 hours of age from the subject's umbilical catheter for caffeine serum concentration measurement by immunoassay to confirm the dosing protocol's achievement of therapeutic and nontoxic concentrations. Subjects were randomized by stratification in blocks of 10 using a computer-generated block randomization schedule. Randomization was performed by a hospital pharmacist. Each infant was assigned a multidigit identification number. As this was a feasibility trial, a convenience sample was set at 10 subjects in each arm, for a recruitment goal of 20 total subjects.

Criteria for intubation were based on previous intubation trials. ${ }^{3}$ Infants were intubated if they had $\mathrm{PCO}_{2}>65 \mathrm{~mm} \mathrm{Hg}$ on any gas within 1 hour of intubation $\mathrm{FiO}_{2}>0.5$ on CPAP to maintain saturation $>88 \%$ for 1 hour or hemodynamic instability (mean arterial blood pressure $<$ GA requiring volume or vasopressor support for $>4$ hours).

All infants had a near-infrared laser sensor (FORE-SIGHT, Casmed, Branford, CT) placed on their anterior forehead as soon as they were clinically stable for continuous cerebral oximetry monitoring. The sensor was placed on the anterior forehead and standardized to minimize effect on readings from different positions. In addition, all infants had four Electrical Cardiometry (EC, Cardiotronic, La Jolla, CA) sensors placed on their head and thorax. EC estimates cardiac output, stroke volume, and other hemodynamic parameters by sending low amplitude, high frequency current through the body and measuring the resulting change in voltage across the thorax. In addition, heart rate and continuous blood pressure (measured from umbilical artery) were recorded every 2 seconds using a bedside data acquisition system.

A single blinded echocardiogram was performed in the first 12 hours of age using the Vivid E9 (GE Healthcare, Wauwatosa, WI) by an investigator (A. C. K) trained in neonatal functional echocardiography. Measures of systemic blood flow including superior vena cava (SVC), left and right ventricular output (LVO, RVO), and diameters of the PDA and PFO were collected on each examination. Shunt direction was also measured. All measures were performed as previously described. ${ }^{11}$ The median inter- and intraobserver variability has been shown to be 18 and $8.1 \%$ for SVC and RVO. ${ }^{12}$

Relevant maternal and neonatal medical information was collected from the electronic medical record and then recorded using the REDCap electronic database application hosted at SHARP Healthcare (REDCap v5.7.1 2014, Vanderbilt University). ${ }^{13}$ Serial measurements of heart rate, cerebral $\mathrm{stO}_{2}$, stroke volume, and mean arterial pressure were averaged over each hour. Normally distributed variables were analyzed with the independent samples $t$-test and nonparametric continuous outcome variables were analyzed with the Mann-Whitney U test. For repeated measurements tests, R ( $\mathrm{R}$ Foundation for 
Statistical Computing, Vienna, Austria, http://www.R-project. org/) and lme4 (Linear mixed-effects models using Eigen and S4, http://arxiv.org/abs/1406.5823) were utilized to perform linear-mixed effects regression (lmer) analysis of the relationship between the respective treatments (i.e., early vs. routine dosage). Demographic data are presented as numbers and proportions for categorical variables or means with standard deviation for normally distributed continuous variables and medians for skewed distribution. Fisher exact test and chi-square test were used to analyze categorical outcome variables. Significance was set at a $p$-value less than 0.05 . For statistics other than the multiple measurements tests, IBM SPSS Statistics 21.0 (SPSS, Inc., Chicago, IL) functions were utilized.

\section{Results}

A total of 21 subjects were enrolled, the final two subjects were twins. Eleven subjects received early caffeine. - Fig. 1 represents a flow diagram that quantifies participant progress through the trial. No parents who were approached for informed consent declined to participate, and no enrolled subjects were lost to follow-up.

There were no significant differences in maternal characteristics or delivery complications between the two groups (-Table 1). Neonates who received early caffeine had a lower incidence of intubation in the first 12 hours (27 vs. 70\%, $p=0.08$ ) and vasopressor requirement in the first 24 hours of age ( 0 vs. $20 \%, p=0.21$ ). Other morbidities were similar between the two groups (-Table 2). Among the outcomes assessed by cardiac ultrasound, SVC flow and RVO were significantly higher in the early caffeine group (-Table $\mathbf{3}$ ). Among the serial measurements taken over the first 12 hours, only blood pressure was significantly different (-Fig. 2). Serum caffeine concentrations sampled after both groups received caffeine (at 13 hours of age) averaged $11.3 \pm 1.3$ $\mathrm{mg} / \mathrm{L}$ in the early group, and $12.2 \pm 2.4 \mathrm{mg} / \mathrm{L}$ in the standard group ( $p=0.33)$.

\section{Discussion}

Caffeine's favorable efficacy and safety record in the NICU for the treatment of apnea of prematurity, and for preventing failed extubation, is well known. ${ }^{4,14-16}$ A potential new direction for caffeine therapy targets early administration for preterm neonates at risk for respiratory failure and cardiovascular insufficiency. Such an intervention should be feasible in most settings as intravenous access is routinely achieved in these patients for provision of intravenous fluids and antibiotics. Infants managed initially on CPAP alone may take time to develop respiratory insufficiency and apnea. Waiting to give caffeine when these symptoms develop may not prevent the need for intubation.

This is the first double blinded randomized controlled trial of caffeine to compare timing of intravenous caffeine therapy. While our study did not demonstrate a difference in the need for endotracheal intubation at 12 hours of age, we did see a trend for decreased intubation by 12 hours of age. To detect a $50 \%$ difference (from 70 to $35 \%$ ), we would need at least 20 infants in each arm to adequately power for this outcome. Our primary hypothesis was based on the assumption that most intubation would occur in the first 12 hours of age.

While not significant, infants receiving routine caffeine had fewer days of mechanical ventilation compared with the early caffeine group (-Table 2 ). This may be due to delayed intubation with worsening pulmonary disease or other negative effects of early caffeine, but need further study in a larger trial.

Our study demonstrated an improvement in systemic blood flow as measured by SVC flow and RVO. SVC flow represents cardiac input and thereby is not affected by the

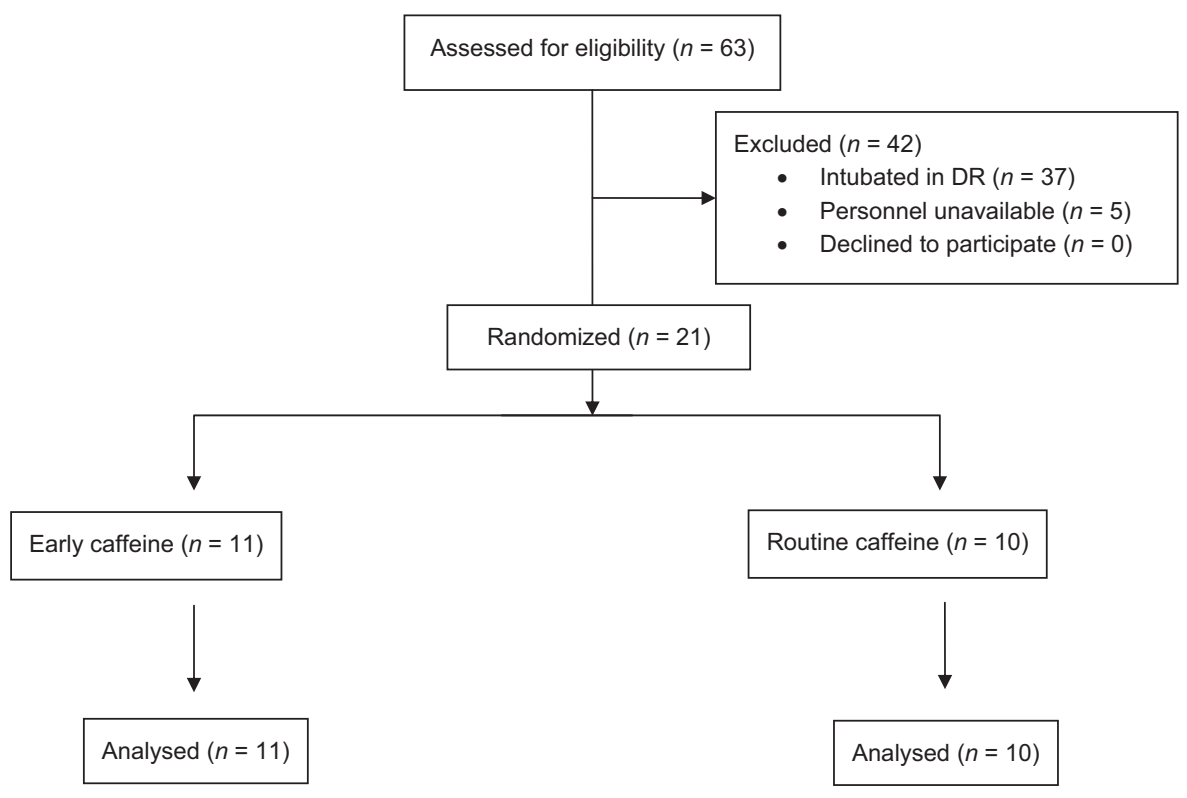

Fig. 1 Consort diagram. 
Table 1 Maternal characteristics

\begin{tabular}{|l|l|l|l|}
\hline Characteristics & $\begin{array}{l}\text { Early } \\
(\boldsymbol{n}=\mathbf{1 1})\end{array}$ & $\begin{array}{l}\text { Routine } \\
(\boldsymbol{n}=\mathbf{1 0})\end{array}$ & $p$-Value \\
\hline Maternal age (y) & $33 \pm 2$ & $30 \pm 2$ & 0.32 \\
\hline Gravida/para & $4 \pm 3$ & $3 \pm 1$ & 0.24 \\
\hline Gestational age (wk) & $27 \pm 0.9$ & $27 \pm 0.9$ & 0.78 \\
\hline Birth weight (g) & $1,007 \pm 169$ & $1,005 \pm 239$ & 0.98 \\
\hline Female (\%) & $6(54)$ & $5(50)$ & 1.0 \\
\hline Antenatal steroids (\%) & $10(91)$ & $10(100)$ & 1.0 \\
\hline Magnesium sulfate (\%) & $10(91)$ & $10(100)$ & 1.0 \\
\hline Neuraxial anesthesia (\%) & $10(91)$ & $9(90)$ & 0.97 \\
\hline C-section delivery (\%) & $8(72)$ & $8(80)$ & 1.0 \\
\hline Labor or uterotonics given (\%) & $7(64)$ & $7(70)$ & 1.0 \\
\hline Premature rupture of membranes (\%) & $6(55)$ & $2(20)$ & 0.18 \\
\hline Chorioamnionitis (\%) & $4(36)$ & $3(30)$ & 1.0 \\
\hline Preeclampsia (\%) & $2(18)$ & $1(10)$ & 1.0 \\
\hline Placental abruption (\%) & $1(9)$ & $2(20)$ & 0.59 \\
\hline
\end{tabular}

Note: Data expressed as means ( \pm standard deviation) unless indicated.

presence of fetal shunts. It is therefore a useful measure of systemic blood flow in the newborn heart. Kluckow et al demonstrated an association between low SVC flow and subsequent development of intraventricular hemorrhage $(\mathrm{IVH}){ }^{12}$ The same investigators previously demonstrated that decreased RVO was closely associated with worsening respiratory disease (defined by $\mathrm{FiO}_{2}$ requirement), severe IVH and death. ${ }^{17}$

We did not demonstrate a difference in left ventricular output by echocardiography or cardiac output measured by electrical impedance. We had previously demonstrated in our preterm infant validation trial that the impedance-derived

Table 2 Neonatal outcomes

\begin{tabular}{|l|l|l|l|}
\hline Outcomes & $\begin{array}{l}\text { Early } \\
(\boldsymbol{n}=\mathbf{1 1})\end{array}$ & $\begin{array}{l}\text { Routine } \\
(\boldsymbol{n}=\mathbf{1 0})\end{array}$ & $p$-Value \\
\hline Median Apgar score 1 min (IQR) & $7(4,8)$ & $7(6,7)$ & 1.0 \\
\hline Median Apgar score 5 min (IQR) & $8(7,8)$ & $8(7,8)$ & 0.49 \\
\hline Required mask ventilation at delivery & $7(64)$ & $7(70)$ & 1.0 \\
\hline Admission arterial blood gas values & \multicolumn{2}{l|}{} \\
\hline $\mathrm{pH}$ & $7.2 \pm 0.1$ & $7.2 \pm 0.1$ & 0.78 \\
\hline PaO $_{2}$ & $63 \pm 18$ & $65 \pm 24$ & 0.77 \\
\hline Paco $_{2}$ & $61 \pm 16$ & $62 \pm 20$ & 0.90 \\
\hline Base deficit & $4.3 \pm 1.9$ & $4.8 \pm 2.9$ & 0.63 \\
\hline Time of caffeine administration (hh:mm) after birth & $1: 12 \pm 0: 28$ & $12: 02 \pm 0: 51$ & $<0.001$ \\
\hline Peak Paco in first 24 h, mm Hg & $67 \pm 14$ & $68 \pm 16$ & 0.81 \\
\hline Required intubation by 12 h & $3(27)$ & $7(70)$ & 0.08 \\
\hline Oxygen at 36 wk corrected & $1(9)$ & $2(20)$ & 0.59 \\
\hline Required inotropes by 24 h & 0 & $2(20)$ & 0.21 \\
\hline Days of mechanical ventilation & $6 \pm 9$ & $3 \pm 5$ & 0.40 \\
\hline Days of oxygen & $32 \pm 26$ & $39 \pm 24$ & 0.66 \\
\hline Intraventricular hemorrhage & 0 & $1(10)$ & 0.48 \\
\hline PDA requiring treatment & $2(18)$ & $4(40)$ & 0.36 \\
\hline
\end{tabular}

Note: Data expressed as means ( \pm standard deviation) unless indicated. 
Table 3 Cardiac ultrasound outcomes

\begin{tabular}{|c|c|c|c|}
\hline Outcomes & $\begin{array}{l}\text { Early } \\
(n=11)\end{array}$ & $\begin{array}{l}\text { Routine } \\
(n=10)\end{array}$ & p-Value \\
\hline Time echocardiogram performed (h) & $6 \pm 4$ & $6 \pm 5$ & 0.87 \\
\hline Superior vena cava flow $(\mathrm{mL} / \mathrm{kg} / \mathrm{min})$ & $101 \pm 25$ & $77 \pm 24$ & 0.04 \\
\hline Right ventricular output (mL/kg/min) & $273 \pm 62$ & $219 \pm 43$ & 0.03 \\
\hline Left ventricular output $(\mathrm{mL} / \mathrm{kg} / \mathrm{min})$ & $214 \pm 68$ & $219 \pm 43$ & 0.48 \\
\hline Patent ductus arteriosus diameter (mm) & $1.4 \pm 1.1$ & $1.7 \pm 0.86$ & 0.59 \\
\hline Atrial shunt diameter (mm) & $1.2 \pm 0.1$ & $1.6 \pm 0.9$ & 0.26 \\
\hline
\end{tabular}

Note: Data expressed as means ( \pm standard deviation).

cardiac output correlated with left ventricular output compared with RVO or SVC flow. ${ }^{18}$ However, the main concern with LVO as a measure of early systemic blood flow during the cardiovascular transition from fetal to newborn life is the extent to which it is confounded by a left to right ductal shunt, which may be particularly large in preterm infants. In the

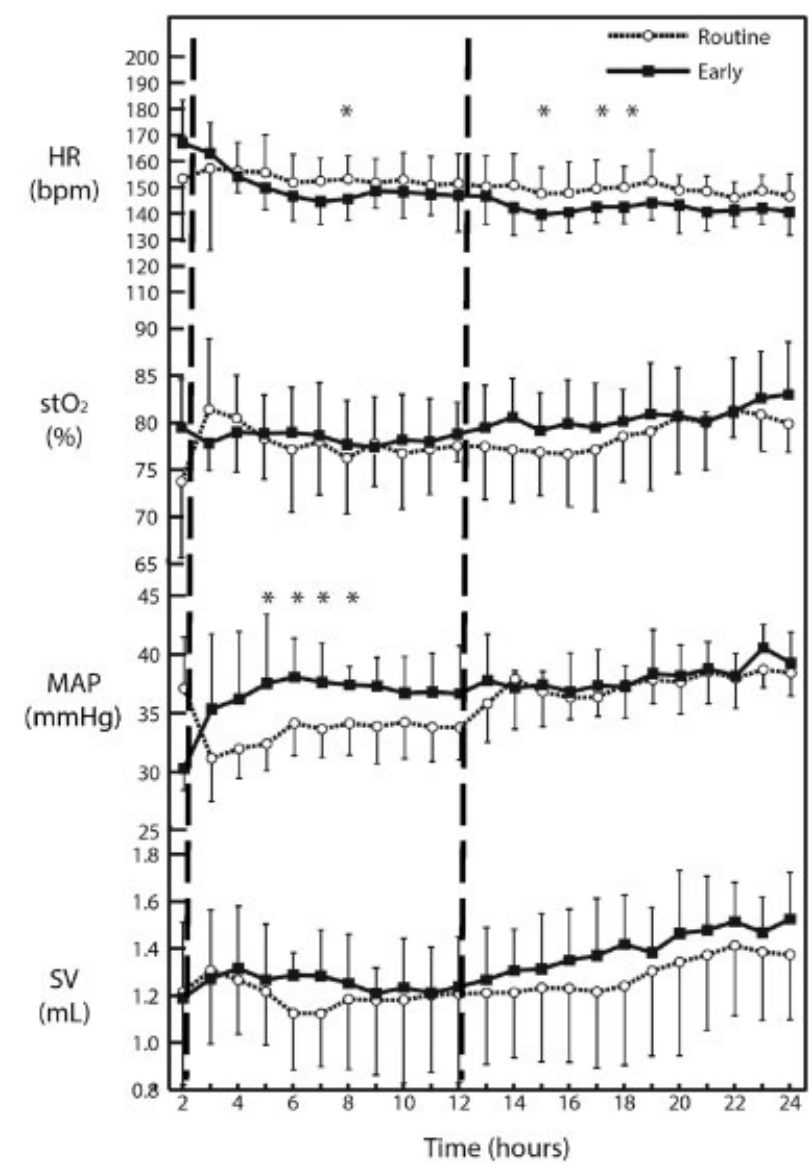

Fig. 2 Continuous heart rate, cerebral saturations $\left(\mathrm{stO}_{2}\right)$, blood pressure (MAP), and stroke volume (SV) ${ }^{*}$ paired $t$-test $=p<0.05$. Blood pressure was significantly higher in the early caffeine group during the first 2 to 12 hours of age (ANOVA $p=0.03$ ). Heart rate (ANOVA $p=0.22$ ), stO2 (ANOVA $p=0.57$ ), and stroke volume (ANOVA $p=0.95$ ) were not significantly different during the same period. Dotted lines indicate timing of caffeine therapy (early or routine). ANOVA, analysis of variance. presence of a ductal shunt, LVO measures both systemic blood flow and the flow across the PDA, and therefore significantly overestimates systemic blood flow. Therefore, it should not be surprising that there was no improvement in cardiac output with caffeine therapy. One prior study has demonstrated an increase of LVO with caffeine, ${ }^{8}$ but others have not demonstrated any change. ${ }^{6,19}$ In each of these studies, caffeine administration was not blinded.

The same observational studies of caffeine have demonstrated higher blood pressures ${ }^{6,8,19}$ and lower measures of cerebral blood flow and saturation as measured by Doppler and near infrared spectroscopy (NIRS). ${ }^{6}$ In our study, infants who received early caffeine therapy had increased in blood pressure in the first 12 hours and a transient decrease in cerebral saturations in both groups as measured by NIRS. The importance of these changes may be critical in the timing of transition particularly for the extremely preterm infant in the first few hours after birth. It is known that caffeine is a potent inhibitor of the vasodilator adenosine. ${ }^{20}$ This action may result in vasoconstriction of cerebral vessels or attenuation of adenosine-induced vasodilation that may occur during hypoxia or hypercarbia. Caffeine may therefore act in a similar mechanism to indomethacin, by decreasing the risk of reperfusion injury by limiting cerebral blood flow. Indomethacin has also demonstrated a transient decrease in cerebral saturations, but within 2 hours of administration was shown to improve both blood pressure and SVC flow. ${ }^{21}$ The protective effects for IVH have only been demonstrated when given shortly after birth. ${ }^{21}$ Avoiding early fluctuations in cerebral blood flow may reduce morbidities, such as IVH, but this needs further study. Interestingly, centers conducting similar retrospective studies of early ( $<3$ days) versus late ( $>3$ days) caffeine are reporting lower rates of IVH in the early caffeine-treated babies. ${ }^{23}$

Patel et al recently described their single center's experience with caffeine given before or after 3 days of age to preterm neonates $\leq 1,250 \mathrm{~g}$ birth weight. ${ }^{24}$ In their retrospective study ( $n=140)$, infants receiving early caffeine were significantly less likely to develop death or BPD (25 vs. 53\%), BPD (24 vs. $51 \%$ ), or a PDA requiring medical or surgical treatment (10 vs. $36 \%$ ). These differences remained statistically significant even after adjustments for confounding variables. In addition, the median duration of endotracheal intubation and mechanical ventilation was more than 2 weeks longer in the late caffeine group (6 vs. 22 days). ${ }^{24} \mathrm{~A}$ 
subsequent multicenter retrospective study of preterm infants with birth weights $<1,500 \mathrm{~g}(n=29,070)$ also demonstrated significant associations between early caffeine initiation before 3 days of age and a reduced incidence of BPD, PDA treatment, and duration of mechanical ventilation. ${ }^{5}$ This study did find an increased rate of death in GA $<24$ week neonates who received early caffeine (33.2 vs. $15.3 \%$ ). However, the authors believed this finding was due to survivor bias. Meanwhile, those in the GA $<24$ week strata who received early caffeine and who survived had significantly lower BPD rates (43.7 vs. 67.1\%) compared with late caffeine recipients. The incidence of late onset sepsis (21.2 vs. $24.5 \%$ ) and inotropic support (21.6 vs $31.6 \%$ ) were also significantly less in the early caffeine group. ${ }^{5}$ Lodha et al with the Canadian Neonatal Network recently reported results from their multicenter retrospective study of caffeine initiation before or after 2 completed days of age in preterm infants GA $<31$ weeks ( $n=5,517)$. Positive outcomes associated with early caffeine in this study included fewer days of mechanical ventilation ( 2 vs. 4 days), a lower incidence of requiring high frequency ventilation on day 2 (6.2 vs. $19.4 \%$ ), a lower odds of having BPD at 36 weeks post menstrual age (odds ratio [OR] 0.79, 95\% confidence interval [CI] 0.64-0.96), and of surgically treated PDA (OR 0.58, 95\% CI 0.42-0.8). Other outcomes such as mortality, necrotizing enterocolitis, retinopathy of prematurity, neurological injury, and total length of hospital stay were not different. The median time of caffeine initiation in this study was 1 day of age in the early group and 4 days of age in the late group. ${ }^{25}$

These studies suggest that early caffeine, more so than caffeine per se, is associated with a reduction in time exposed to endotracheal intubation and positive pressure or mechanical ventilation, which in turn reduces the risk of developing BPD and possibly other associated complications. While it is natural to assume that the respiratory and cardiovascular stimulant and vasoconstrictive effects of caffeine provides the pharmacologically plausible basis for these observed outcomes, recent animal studies indicate that caffeine can also ameliorate the inflammatory response and lung injury due caused by acute hyperoxia or intrauterine infection. ${ }^{26,27}$

Our study has several limitations. First, since this was a pilot study, we were underpowered to achieve any differences in the outcome of reducing intubation. Until a larger randomized controlled trial is completed our clinical outcomes can only be speculative. Second, given that our study included only nonintubated infants at birth, it is unclear whether early caffeine would have the same hemodynamic effects on potentially sicker intubated infants. Given the improved measures of hemodynamics it may be prudent to include these infants in future trials of caffeine.

In conclusion, this pilot study demonstrated that conducting a prospective, randomized, placebo-controlled trial comparing early to late caffeine administration in extremely preterm neonates is feasible. We found that giving early intravenous caffeine administration to nonintubated, extremely low GA neonates resulted in improved systemic blood flow, blood pressure, and cerebral oxygenation during early neonatal life. Larger prospective studies are needed to determine the effects of early caffeine on the need for intubation, IVH, and related long-term outcomes such as chronic lung disease and neurodevelopmental impairment.

\section{Acknowledgments}

The investigators were supported with seed funding from the Tarsadia Foundation and the Little Giraffe Foundation. The authors would like to acknowledge Kathy Arnell, Debra Petruzzelli, Jane Steen, Debbie Dennington, and Randy Noprasi of the Neonatal Research Institute, the Advance Life Support nurses and pharmacists of the neonatal intensive care unit, and San Diego Neonatology physicians, at Sharp Mary Birch Hospital for Women and Newborns for their help in enrollment, set up, and placement of monitoring equipment.

\section{References}

1 Committee on Fetus and Newborn; American Academy of Pediatrics. Respiratory support in preterm infants at birth. Pediatrics 2014;133(1):171-174

2 Morley CJ, Davis PG, Doyle LW, Brion LP, Hascoet JM, Carlin JB; COIN Trial Investigators. Nasal CPAP or intubation at birth for very preterm infants. N Engl J Med 2008;358(7):700-708

3 Finer NN, Carlo WA, Walsh MC, et al; SUPPORT Study Group of the Eunice Kennedy Shriver NICHD Neonatal Research Network. Early CPAP versus surfactant in extremely preterm infants. N Engl J Med 2010;362(21):1970-1979

4 Schmidt B, Roberts RS, Davis P, et al; Caffeine for Apnea of Prematurity Trial Group. Caffeine therapy for apnea of prematurity. N Engl J Med 2006;354(20):2112-2121

5 Dobson NR, Patel RM, Smith PB, et al. Trends in caffeine use and association between clinical outcomes and timing of therapy in very low birth weight infants. J Pediatr 2014;164(5):992-998.e3

6 Tracy MB, Klimek J, Hinder M, Ponnampalam G, Tracy SK. Does caffeine impair cerebral oxygenation and blood flow velocity in preterm infants? Acta Paediatr 2010;99(9):1319-1323

7 Soloveychik V, Bin-Nun A, Ionchev A, Sriram S, Meadow W. Acute hemodynamic effects of caffeine administration in premature infants. J Perinatol 2009;29(3):205-208

8 Walther FJ, Erickson R, Sims ME. Cardiovascular effects of caffeine therapy in preterm infants. Am J Dis Child 1990;144(10):1164-1166

9 Kribs A, Vierzig A, Hünseler C, et al. Early surfactant in spontaneously breathing with nCPAP in ELBW infants-a single centre four year experience. Acta Paediatr 2008;97(3):293-298

10 Aguar M, Cernada M, Brugada M, Gimeno A, Gutierrez A, Vento M. Minimally invasive surfactant therapy with a gastric tube is as effective as the intubation, surfactant, and extubation technique in preterm babies. Acta Paediatr 2014;103(6):e229-e233

11 Katheria A, Leone T. Altered transitional circulation in infants of diabetic mothers with strict antenatal obstetric management: a functional echocardiography study. J Perinatol 2012;32(7): 508-513

12 Kluckow M, Evans N. Low superior vena cava flow and intraventricular haemorrhage in preterm infants. Arch Dis Child Fetal Neonatal Ed 2000;82(3):F188-F194

13 Harris PA, Taylor R, Thielke R, et al. Research electronic data capture (REDCap) - A metadata-driven methodology and workflow process for providing translational research informatics support. J Biomed Inform 2009;42(2):377-381

14 Henderson-Smart DJ, Davis PG. Prophylactic methylxanthines for endotracheal extubation in preterm infants. Cochrane Database Syst Rev 2010;(12):CD000139 
15 Steer P, Flenady V, Shearman A, et al; Caffeine Collaborative Study Group Steering Group. High dose caffeine citrate for extubation of preterm infants: a randomised controlled trial. Arch Dis Child Fetal Neonatal Ed 2004;89(6):F499-F503

16 Erenberg A, Leff RD, Haack DG, Mosdell KW, Hicks GM, Wynne BA. Caffeine citrate for the treatment of apnea of prematurity: a double-blind, placebo-controlled study. Pharmacotherapy 2000; 20(6):644-652

17 Evans N, Kluckow M. Early determinants of right and left ventricular output in ventilated preterm infants. Arch Dis Child Fetal Neonatal Ed 1996;74(2):F88-F94

18 Song R, Rich W, Kim JH, Finer NN, Katheria AC. The use of electrical cardiometry for continuous cardiac output monitoring in preterm neonates: a validation study. Am J Perinatol 2014;31(12): $1105-1110$

19 Hoecker C, Nelle M, Poeschl J, Beedgen B, Linderkamp O. Caffeine impairs cerebral and intestinal blood flow velocity in preterm infants. Pediatrics 2002;109(5):784-787

20 Smits P, Lenders JW, Thien T. Caffeine and theophylline attenuate adenosine-induced vasodilation in humans. Clin Pharmacol Ther 1990;48(4):410-418

21 Lemmers PM, Toet MC, van Bel F. Impact of patent ductus arteriosus and subsequent therapy with indomethacin on cerebral oxygenation in preterm infants. Pediatrics 2008;121(1):142-147
22 Schmidt B, Asztalos EV, Roberts RS, Robertson CM, Sauve RS, Whitfield MF; Trial of Indomethacin Prophylaxis in Preterms (TIPP) Investigators. Impact of bronchopulmonary dysplasia, brain injury, and severe retinopathy on the outcome of extremely lowbirth-weight infants at 18 months: results from the trial of indomethacin prophylaxis in preterms. JAMA 2003;289(9): 1124-1129

23 Gupta D, Gupte A, Chouthai ND. Effect of early caffeine on the neurodevelopmental outcome of very low birth weight newborns: A cohort study. EPAS 2014;1555:792

24 Patel RM, Leong T, Carlton DP, Vyas-Read S. Early caffeine therapy and clinical outcomes in extremely preterm infants. J Perinatol 2013;33(2):134-140

25 Lodha A, Seshia M, McMillan DD, et al; for the Canadian Neonatal Network. Association of early caffeine administration and neonatal outcomes in very preterm neonates. JAMA Pediatr 2014 (e-pub ahead of print). doi: 10.1001/jamapediatrics.2014.2223

26 Weichelt U, Cay R, Schmitz T, et al. Prevention of hyperoxiamediated pulmonary inflammation in neonatal rats by caffeine. Eur Respir J 2013;41(4):966-973

27 Köroğlu OA, MacFarlane PM, Balan KV, et al. Anti-inflammatory effect of caffeine is associated with improved lung function after lipopolysaccharide-induced amnionitis. Neonatology 2014; 106(3):235-240 